\title{
Coronary Artery Disease
}

National Cancer Institute

\section{Source}

National Cancer Institute. Coronary Artery Disease. NCI Thesaurus. Code C26732.

Narrowing of the coronary arteries due to fatty deposits inside the arterial walls. The diagnostic criteria may include documented history of any of the following: documented coronary artery stenosis greater than or equal to 50\% (by cardiac catheterization or other modality of direct imaging of the coronary arteries); previous coronary artery bypass surgery (CABG); previous percutaneous coronary intervention $(\mathrm{PCI})$; previous myocardial infarction. (ACC) 